\title{
Articulación entre tecnología y producción: Los contratistas de labores en la región pampeana*
}

\author{
Patricia Beatriz Lombardo,' Mábel García² \\ Universidad de Buenos Aires (Argentina)
}

Recibido: diciembre 10 de 2014 - Revisado: enero 9 de 2015 - Aceptado: mayo 20 de 2015

Referencia formato APA: Lombardo, P. B., \& García, M. (2015). Articulación entre tecnología y producción: Los contratistas de labores en la región pampeana. Revista Cientifica Guillermo de Ockham, 13(1), 79-86.

\section{Resumen}

En el marco de las profundas transformaciones que se produjeron a partir de la segunda mitad del siglo XX en la región pampeana, el contratista de labores agrícolas tomó una nueva dimensión gracias a su capacidad para responder de forma ágil y flexible a los cambios técnicos y organizativos. Este sujeto social ocupa una posición central en la escena productiva, articula la oferta y la demanda de tecnología y se configura en una pieza clave para el aumento de la eficiencia productiva. El artículo tiene como objetivo avanzar en el análisis referido a la caracterización del contratista en cuanto a su relevancia socioeconómica, tecnológica y productiva. Se utilizó una estrategia metodológica basada en el relevamiento de información primaria a través de encuestas a contratistas que, a su vez, son productores agropecuarios. Dicho instrumento se diseńó para indagar sobre una serie de variables que permitieron caracterizar y diferenciar dos tipos de contratistas según su actividad económica principal: los contratistas productores y los productores contratistas. Los principales resultados obtenidos, dan cuenta de la heterogeneidad de estos sujetos y del grado de complejidad alcanzado.

Palabras clave: Sujeto social, cambio tecnológico, productividad, pluriactividad, heterogeneidad

\section{Relationships between technology and agricultural production: the contractor of labors in the Argentine Pampas}

\section{Abstract}

In the setting of the deep transformations that took place during the second half of the XX century in the Argentine Pampas, the contractor of agricultural labors reached a new dimension because of his ability to respond agilely and flexibly to technical and organizational changes. This social subject holds a main position in the productive scene articulating the supply and demand of technology, and becoming a key element for the increase of productive efficiency.

* Este artículo se deriva del proyecto de investigación Cambio tecnológico y producción: un análisis de la figura del contratista como agente articulador, financiado por la Universidad de Buenos Aires en el marco de la programación científica 2011-2014. Fecha de inicio: 1º julio de 2011. Fecha de finalización: 30 de junio de 2014. Código: 20020100100481.

1. Ingeniera Agrónoma de la Universidad Nacional de Mar del Plata. Doctora de la Universidad de Buenos Aires, con mención en el área de educación. Profesora asociada e investigadora de la Facultad de Agronomía (cátedra de Economía Agraria) de la Universidad de Buenos Aires. E-mail: patricia@ agro.uba.ar.

2. Ingeniera Agrónoma de la Universidad de Buenos Aires. Magíster Science en Ingeniería Ambiental de la Universidad Tecnológica Nacional. Profesora adjunta e investigadora de la Facultad de Agronomía (cátedra de Economía Agraria) de la Universidad de Buenos Aires. E-mail: mabelg@agro.uba.ar. 
The objective of the paper is to advance in the referred analysis to the characterization of the contractor regarding his or her socioeconomic, technological, and productive importance. A methodological strategy based on the study of primary information was used through surveys done among contractors who are producers as well. The instrument mentioned above was designed to examine a group of variables that allowed us to characterize and differentiate two types of contractors according to their economic activity: the ones who are mainly contractors and others whose principal activity is the agriculture production. The main results of this analysis show the heterogeneity of these social subjects and the degree of complexity reached.

Keywords: Social subject, technological change, productivity, multiple activity and heterogeneity

\section{Articulação entre tecnologia e produção: trabalho empreiteiro nos Pampas Argentina}

\section{Resumo}

No contexto das profundas mudanças que ocorreram a partir da segunda metade do século XX, na regiāo dos Pampas, a agricultura contratante atingiu uma nova dimensão, devido à sua capacidade de responder rapidamente e de forma flexível às mudanças técnicas e organizacionais. Este sujeito social ocupa um lugar central no cenário produtivo articular a oferta ea procura de tecnologia e configurada como uma chave para aumentar a eficiência da produção. $\mathrm{O}$ artigo tem como objetivo fazer avançar a análise baseada na caracterização do contratante em termos de sua relevância sócio-econômica, tecnológica e produtiva. A estratégia metodológica baseada em um levantamento de dados primários através de inquéritos aos contratantes que, por sua vez, os agricultores são usados. Este instrumento foi concebido para investigar uma série de variáveis que permitiram caracterizar e diferenciar dois tipos de contratantes de acordo com as suas principais produtores de atividade econômica empreiteiros e produtores empreiteiros. Os principais resultados obtidos, explicar a heterogeneidade desses temas e grau de complexidade alcançado.

Palavras-chave: Sujeito social, mudança tecnológica, a produtividade, a atividade múltipla e heterogeneidade.

\section{Introducción}

El contratista de labores agrícolas ha desempeñado, históricamente, un papel significativo en la expansión e intensificación de la actividad agrícola en la región pampeana. ${ }^{3}$ Constituye una modalidad de trabajo que acompaña el desarrollo de la agricultura cerealera extensiva desde sus inicios, principalmente a través de los contratistas de trilla (Pucciarelli, 1998).

El contratista es un sujeto social que brinda servicios a terceros de laboreo, siembra, protección de cultivos y cosecha, y recibe como pago por su prestación dinero o especies. Considerado como un articulador entre la oferta y la demanda de tecnología el contratista se ha configurado como una pieza clave para el aumento de la eficiencia productiva. Con la generalización de niveles tecnológicos más avanzados este actor ha incrementado su importancia (Tort, 1983).

La difusión y consolidación de esta figura representa el reverso de los problemas acarreados por cuestiones referidas a formas de distribución y acceso a la tierra que mostró el sector agropecuario. Gallo-Mendoza (1970), considera que en el caso argentino, el resultado manifiesto en los predios de las formas de tenencia no propietarias -esencialmente arrendatarios y aparceros- es la descapitalización en mejoras fijas y una sobremecanización, cuyo excedente integra el capital de los contratistas.

El proceso de modernización agrícola, puso en el centro de la escena productiva al contratista. Su ascenso se vio favorecido por el hecho de que, en los años iniciales de este proceso, algunos pequeños y medianos productores adquirieron una cantidad de maquinaria mayor a la que

3. Es una región conformada por las provincias de Buenos Aires, Entre Ríos, Santa Fe, Córdoba, La Pampa y San Luis. Es una extensa llanura, con ondulaciones, clima templado y considerada una de las más fértiles del mundo. Es la región más importante del territorio argentino, puesto que sus características de clima y suelo la transforman en una zona agrícola y ganadera por excelencia. 
necesitaba para cultivar sus tierras. De este modo, lograron vender servicios a otros productores ampliando así las posibilidades de producción e impulsando la innovación técnica vinculada con la maquinaria agrícola (Piñeiro \& Villarreal, 2005).

Varios factores aumentaron la proporción de los trabajos cortos temporarios por sobre las tareas permanentes o prolongadas, entre ellos: el sistema de siembra directa, los herbicidas que reemplazaron labores mecánicas, las semillas genéticamente modificadas, la capacidad de los nuevos tractores y las cosechadoras.

Esta reducción de los tiempos de trabajo también posibilitó un mayor protagonismo del contratismo de servicios, las labores podían ser contratadas y ejecutadas por empresas externas en un lapso breve, sin necesidad de adquirir toda la maquinaria, reforzando de esta manera la tendencia a dejar la residencia rural. Para quienes estaban en posesión del equipamiento, las tareas breves los liberaron de una masa de tiempo, excedente que podían utilizar para prestar servicios fuera de su explotación (Villulla $\&$ Amarilla, 2011).

A partir de la década de 1990, con el surgimiento de los pooles de siembra ${ }^{4}$ se abrió para los contratistas una significativa posibilidad de crecimiento que los llevó a convertirlos en importantes protagonistas del negocio agrícola (Beltrán, 2007). Garbers (2009) remarca que la concertación de acuerdos entre pooles y contratistas es tendencia creciente.

Estos sujetos sociales han adquirido una nueva dimensión y presencia (Slutzky, 2009), que los faculta para responder de manera ágil y flexible a los cambios técnicos y de organización laboral. Cambios que están en consonancia con una serie de nuevas situaciones y readaptaciones a fin de responder a las exigencias que reclama el medio, mejorando así su parque de maquinaria, su capacidad de trabajo y sus conocimientos (Agüero, Rivarola $\&$ Maldonado, 2007).

Los cambios tecnológicos evidenciados en el agro fueron en parte, canalizados hacia los productores por el contratista, quien especializado en la tenencia de un factor productivo (maquinaria), conjuga capacidad empresarial y financiera, necesidad de aplicar los cambios tecnológicos junto con conocimiento tácito y posibilidad de diversificar el riesgo agropecuario, amén de mantener estrechas relaciones con el resto de los actores (Lódola, 2008).
Uno de los principales factores que propiciaron la consolidación del contratismo rural es la demanda de una mecanización especializada que no pudo ser afrontada por gran parte de los productores agropecuarios, ya sea por la escala reducida de sus predios o por lo elevado de la inversión requerida (Bustamante \& Maldonado, 2008).

El contratismo ha facilitado la continuidad de productores agropecuarios pequeños (Azcuy-Ameghino \& Fernández, 2008), no solo porque parte de ellos hacen sus tareas mediante el uso de servicios mecanizados contratados, sino porque el resto se dedica, precisamente, a vender tales servicios. Lo anterior puede encuadrarse dentro del denominado efecto permanencia, que se corresponde con situaciones de contratación parcial de labores (Zulian, 2010).

El contratismo se asocia a la generación de una actividad "refugio" para aquellos productores "expulsados" por el modelo de producción. Por el otro, el contratista rural y su quehacer dentro del agro -en los últimos años- han contribuido para que las unidades agropecuarias del tipo productivo-domésticas (con baja dotación de tierra y capital) aumenten sus beneficios a través de la elevación de los niveles de productividad y rentabilidad.

Si bien aquellos productores con maquinaria propia la siguen utilizando, la terciarización de las labores es una modalidad cada vez más difundida entre los distintos tipos de productores. Mientras que a las empresas agropecuarias -de mayor escala- y a los pooles de siembra les permite mantener su flexibilidad productiva y económica, a los agricultores familiares les posibilita la permanencia (Muzlera, 2010).

Este artículo, enmarcado en un proyecto de investigación acreditado y financiado por la Universidad de Buenos Aires, tiene como objetivo analizar las principales características socioeconómicas, tecnológicas y productivas de los contratistas de labores agrícolas, quienes a su vez son productores agrarios que residen en distintas zonas de la región pampeana.

\section{Estrategia metodológica}

En este trabajo se han utilizado reprocesamientos especiales del Censo Nacional Agropecuario 2008 (CNA, 2008) que contribuyen a dimensionar el contratismo de servicios de maquinaria en la región pampeana.

4. En Argentina, es la denominación que recibe un sistema de producción agraria caracterizado por el papel determinante del capital financiero y la organización de un sistema empresarial transitorio que asume el control de la producción mediante el arrendamiento de grandes extensiones de tierra y la contratación de maquinarias, con el objetivo de generar economías de escala y altos rendimientos. 
La magnitud y complejidad del universo de estudio (los contratistas de labores agrícolas de la región pampeana) dificulta que este sea abordado en su totalidad. Por lo tanto, se optó por seleccionar algunas zonas de la región pampeana y definir una muestra intencional basada en un conjunto de atributos considerados relevantes en las unidades de estudio. Se debe tener en cuenta que este tipo de muestreo es no probabilístico, por lo tanto no se pueden hacer generalizaciones por la falta de certeza acerca de la representatividad de la muestra. Se utilizó una estrategia metodológica basada en el relevamiento de información primaria mediante encuestas a contratistas residentes -que, a su vez son productores- $y$ desarrollan su actividad en partidos o departamentos de las distintas provincias que componen la región pampeana (Buenos Aires, Córdoba y Entre Ríos).

Dichas encuestas revelaron información sobre las siguientes variables: origen de la actividad de contratista, superficie operada (como prestador y como productor) y labores que lleva a cabo como contratista. Se especifican forma de pago y modalidad de contrato, organización laboral, dimensionamiento del parque de maquinaria y antigüedad, inversiones realizadas vinculadas a la actividad de prestación de servicios de maquinarias y formas de financiamiento y participación en formas asociativas.

La información analizada en este artículo, constituye un resultado parcial de una investigación en marcha y representa un avance en la caracterización de estos sujetos sociales que desempeñan un papel significativo como articuladores entre el cambio tecnológico y la producción.

\section{Presentación de los resultados}

\section{La información censal y los contratistas de servicios de maquinaria}

$\mathrm{Al}$ analizar la información proveniente del reprocesamiento del Censo Nacional Agropecuario 2008 (CNA 2008), se observa que el $40 \%$ de la superficie agropecuaria total de la región pampeana es trabajada -en alguna medida- por contratistas de servicios de maquinaria. Las variaciones se presentan según las provincias. Así, Buenos Aires y Córdoba se encuentran por encima de este porcentaje (51\% y $48 \%$ respectivamente) mientras que Entre Ríos, La Pampa y Santa Fe están por debajo (26\%, $22 \%$ y $36 \%$ respectivamente) (Tabla 1). Estos servicios de maquinarias son brindados por otros productores, empresas de servicios de maquinaria y organismos públicos.
Tabla 1

Relación entre superficie agropecuaria total y superficie contratada. Región pampeana, 2008

\begin{tabular}{cccc}
\hline Provincia & $\begin{array}{c}\text { Superficie total } \\
\text { (en ha.) }\end{array}$ & $\begin{array}{c}\text { Superficie } \\
\text { contratada } \\
\text { (en ha.) }\end{array}$ & Porcentaje \\
\hline Buenos Aires & 19.547 .064 & 10.032 .551 & $51 \%$ \\
\hline Córdoba & 11.901 .299 & 5.701 .018 & $48 \%$ \\
\hline Entre Ríos & 5.967 .936 & 1.574 .771 & $26 \%$ \\
\hline La Pampa & 12.251 .327 & 2.680 .524 & $22 \%$ \\
\hline Santa Fe & 10.748 .052 & 3.881 .408 & $36 \%$ \\
\hline Total & 60.415 .678 & 23.870 .271 & $40 \%$ \\
\hline
\end{tabular}

Fuente: Elaboración propia con datos del CNA 2008, Indec.

Si se centra la atención en la superficie de servicios de maquinaria prestados por productores agropecuarios y declarada por estos, se observa que solo el $32 \%$ del total de la superficie contratada a nivel regional, es trabajada por prestadores productores y el restante $68 \%$, principalmente, por empresas de servicios de maquinaria (Tabla 2).

Tabla 2

Participación de los contratistas productores sobre el total de superficie contratada

\begin{tabular}{ccc}
\hline Provincia & Sup. contratada (ha) & Prestadores \\
\hline Buenos Aires & 10.032 .551 & $26 \%$ \\
\hline Córdoba & 5.701 .018 & $30 \%$ \\
\hline Entre Ríos & 1.574 .771 & $41 \%$ \\
\hline La Pampa & 2.680 .524 & $18 \%$ \\
\hline Santa Fe & 3.881 .408 & $57 \%$ \\
\hline Total & 23.870271 & $32 \%$ \\
\hline
\end{tabular}

Fuente: Elaboración propia con datos del CNA 2008, Indec.

En la siguiente tabla se presenta la información correspondiente a la cantidad de explotaciones agropecuarias (EAP) que prestan servicios de maquinaria para la ejecución de algún tipo de labor así como la superficie trabajada.

Tabla 3

Cantidad de EAP que prestan servicios de maquinarias y superficie trabajada. Región pampeana, 2008

\begin{tabular}{ccc}
\hline Provincia & EAP & Superficie (ha) \\
\hline Buenos Aires & 1269 & 2.608 .096 \\
\hline Córdoba & 1047 & 1.711 .534 \\
\hline Entre Ríos & 378 & 639.548 \\
\hline La Pampa & 230 & 486.702 \\
\hline Santa Fe & 1403 & 2.209 .638 \\
\hline Total & 4327 & 7.655 .518 \\
\hline
\end{tabular}


Un estudio de García et al. (2010), muestra la vinculación entre las EAP -según deciles de superficie agropecuaria-y cada tipo de prestador. Se observa que las empresas de servicios se vinculan, en mayor medida, con los grandes productores, mientras que los prestadores productores de servicios lo hacen con las EAP más pequeñas.

\section{Análisis de las encuestas realizadas a contratistas de servicios de maquinaria}

Se hicieron encuestas a treinta y un contratistas de servicios de maquinaria, que en conjunto trabajan una superficie de 111.695 ha. Estos residen y prestan servicios en distintas provincias que componen la región pampeana argentina (Buenos Aires, Córdoba y Entre Ríos) y desarrollan su actividad en localidades comprendidas dentro de los partidos/departamentos donde residen y solo algunos en partidos/departamentos vecinos.

Todos los contratistas encuestados comparten dicha actividad con la de productor agropecuario. Un $52 \%$ de estos considera la prestación de servicios como un complemento de la actividad productiva y para el $48 \%$ restante constituye su actividad principal. A su vez, un $19 \%$ lleva a cabo otras actividades como asesoramiento técnico o venta de agroinsumos, las cuales resultan un complemento del servicio prestado y permiten diversificar aún más sus fuentes de ingresos extraprediales.

Los momentos de inicio como productores van desde 1960 en adelante, en tanto las tareas de prestación de servicios de maquinarias comienzan en su mayor parte durante la década de 1990, como emprendimientos en los que participan distintos integrantes de la familia.

En cuanto a la decisión de incursionar en la prestación de servicios, se destacan razones vinculadas a la posibilidad de incrementar los ingresos, ya sea para lograr un mejor aprovechamiento de la inversión en maquinaria y equipos, o bien para aumentar la escala de producción. En algunos casos, si bien la escala de producción les permite el sostenimiento familiar, la prestación de servicios no solo significa un ingreso adicional, sino también la posibilidad de tecnificarse y mejorar, a su vez, la calidad de los servicios prestados.

Con respecto al tipo jurídico adoptado e independientemente de la actividad principal (productor o contratista), predominan las personas físicas (71 \%) y en menor medida (29\%), las formas societarias más complejas (sociedades comerciales), principalmente sociedades anónimas y de responsabilidad limitada. No se identificó relación alguna entre la complejidad de la organización societaria y el tipo de actividad desarrollada como contratista.

En aquellos casos en los que la actividad principal es la de productor, el rango de superficie trabajada para terceros varía entre las 400 ha y las 3.000 ha. En los casos donde prima la prestación de servicios la superficie trabajada oscila entre las 1.200 ha y 7.900 ha. Los primeros operan una superficie por cuenta propia como productores que varía entre las 150 ha y las 3.000 ha; en cambio, los segundos no superan las 1.200 ha.

El régimen de tenencia de la tierra operada por cuenta propia, es una característica que diferencia a los contratistas analizados. Los productores contratistas (PC) se caracterizan por usos de tierras en forma pura o combinada con alguna forma de alquiler (arrendamiento o contrato accidental); esto con el propósito de incrementar su escala de producción. Entre los contratistas productores (CP) predomina el arrendamiento en forma pura y en combinación con propiedad.

Los servicios prestados por los CP están concentrados en tres labores estrechamente vinculadas con el modelo tecnológico vigente y difundido: la siembra directa, la pulverización y la cosecha de granos. Un $70 \%$ de estos contratistas prestan servicios referidos a dos o más de estas labores y el resto se especializa en una sola. Dentro de este grupo se destacan dos situaciones: a. algunos han extendido su radio de acción a partidos vecinos, $y \mathrm{~b}$. otros prestan servicios casi en forma exclusiva a ciertos clientes que tienen como característica su gran escala de producción.

Por su parte, los PC prestan servicios más diversificados -incluso algunos que no son provistos por los $\mathrm{CP}$ - esto se debe a que tienen parques de maquinaria completos. El $100 \%$ presta servicios de maquinaria relacionados con labranza y labores complementarias de preparación del suelo, que se complementan con una o más de las siguientes: siembra convencional, siembra directa, cuidados culturales mecánicos, pulverización terrestre, cosecha de granos y preparación de reservas forrajeras. Una particularidad de estos prestadores radica en que priorizan las tareas dentro de sus propios establecimientos y la prestación de servicios está orientada a usuarios dentro de los partidos o departamentos donde residen (generalmente vecinos).

Todos los encuestados coinciden en que establecen arreglos informales ("de palabra") con sus clientes y que la retribución estipulada por los servicios prestados consiste en una tarifa fija en dinero. Las diferencias residen en la forma de pago del servicio: un $39 \%$ combina el pago por 
campaña agrícola y por labor, un $36 \%$ exclusivamente por campaña y un $25 \%$ solo por labor.

Es importante resaltar que el modo de vinculación (arreglo informal) evidencia un conocimiento previo basado, principalmente, en relaciones de vecindad geográfica que en ocasiones permite llevar a cabo acuerdos específicos entre las partes.

La prestación de servicios en los PC se sustenta, principalmente, en la mano de obra familiar (sin remuneración fija) -incluido el productor-complementada en algunos casos por personal permanente o personal contratado en forma directa. A diferencia de los PC, los CP ocupan una mayor cantidad de personal para la prestación de servicios, casos en los cuales prima la mano de obra asalariada (personal permanente o contratado en forma directa o indirecta).

Los parques de maquinarias declarados por los $\mathrm{CP}$ que se especializan en la prestación de labores de siembra directa, pulverización y cosecha de granos, se restringen solo a las maquinarias necesarias para su cumplimiento.

Si bien los parques de maquinarias que tienen los PC son heterogéneos, tanto por las maquinarias adquiridas como por las dimensiones alcanzadas. La mayoría de ellos tiene al menos una sembradora de siembra directa, una pulverizadora y una cosechadora, lo que constituye la maquinaria básica que requiere el modelo tecnológico difundido y profundizado a partir de los ańos noventa (soja transgénica, glifosato y siembra directa).

En su mayoría, las maquinarias de ambos tipos de contratistas tienen una antigüedad inferior a los diez ańos, lo cual indica parques de maquinarias modernos y actualizados acordes con los adelantos tecnológicos registrados en la última década.

En los últimos cinco años, los contratistas relevados han efectuado inversiones en maquinarias y equipos. El $66 \%$ de las maquinarias han sido compradas en estado nuevo y el resto usadas (predomina el origen nacional). Se notan diferencias entre $\mathrm{PC}$ y $\mathrm{CP}$ respecto del tipo y cantidad de maquinarias adquiridas, así como de los montos de las inversiones. Mientras que los primeros han adquirido en promedio dos maquinarias, los $\mathrm{CP}$ adquirieron cuatro. No se registran inversiones en bienes inmuebles, lo cual plantea el interrogante sobre los espacios disponibles para el resguardo de las maquinarias y equipos, así como para llevar a cabo su mantenimiento y reparación.

Para el análisis de la financiación, es necesario distinguir entre el financiamiento destinado a cubrir el capital de trabajo (gastos corrientes para la prestación del servicio) y el de inversión. El 100 \% de los encuestados financia el capital de trabajo con recursos propios.

Con respecto al capital de inversión, el $75 \%$ de los PC combinan -en distinta proporción- recursos propios y financiamiento bancario proveniente de la banca pública -principalmente provincial- y el $25 \%$ restante financia sus inversiones con recursos propios exclusivamente. En el caso de los CP, todos utilizan distintas fuentes de financiamiento: el $66 \%$ combina recursos propios con banca pública -tanto nacional como provincial-y el resto complementa la combinación anterior (recursos propios con banca pública) con la financiación proveniente de concesionarias de maquinarias.

Por último, con respecto a su participación como miembros en organizaciones que reúnen a prestadores de servicios, todos los encuestados respondieron en forma negativa.

\section{Discusión de los resultados presentados}

Si bien el universo de contratistas de servicios de maquinaria analizado es sumamente acotado, la información revelada pone de manifiesto lo observado anteriormente por otros autores (Preda, 2000; González, Román \& Blanchard, 2001; Retamoza, 2001; Bustillo, 2004), sobre la complejidad de la figura de este sujeto social. Este no solo se puede vincular con pequeños y medianos sobremecanizados, sino también con otros caracterizados por un mayor nivel de capitalización, implicando de este modo diferencias en los objetivos que persiguen, en su estrategia productiva, en su capacidad económico-financiera, en su organización laboral y en el nivel de profesionalización alcanzado.

Durante la década de los noventa, la región pampeana estuvo signada por una serie de transformaciones productivas, económicas y sociales entre las que destaca la difusión de una serie de innovaciones tecnológicas destinadas a aumentar la productividad de las unidades productivas y por ende, su competitividad. En este contexto, la figura del contratista cobra gran relevancia como forma de conexión entre el capital, la tierra y el trabajo. Los servicios brindados por estos se han configurado como piezas claves dentro del desempeño global del sector.

La conjunción de siembra directa y semilla transgénica constituyó un cambio cualitativo en el modelo tecnológico que modificó la estructura del capital fijo y variable y simplificó aún más la dotación de maquinarias necesarias (De Nicola, 2003). Sin embargo, un número importante 
de productores no disponía de máquina pulverizadora, cosechadora y sembradora de siembra directa, ya sea por conveniencia económica o por no disponer del capital necesario para adquirirla. Esta situación se vio compensada gracias a una amplia oferta del servicio de pulverización, cosecha y siembra directa, con máquinas de gran capacidad y calidad de trabajo.

Fue precisamente durante la década de los noventa que se promovió la incorporación de tecnología para aumentar la eficiencia y la rentabilidad. La mayoría de los encuestados comienza su actividad como contratista y estructuran emprendimientos que se constituyen en una fuente de ingreso extrapredial que alcanza trascendencia en función de los casos. Esta situación es consecuencia de una sobremecanización de los productores que los lleva a ampliar sus tareas y utilizar sus máquinas en campos vecinos y en otras zonas de la región (Agüero et al., 2007).

La prestación de servicios a terceros por parte de algunos titulares de unidades productivas, es una de las formas difundidas de las actividades extraprediales que, de este modo, amplían sus ingresos mediante un uso más intensivo del parque de maquinarias de que disponen (Azcuy-Ameghino \& Fernández, 2008).

Con base en la información, se identificaron similitudes y diferencias que contribuyen a su caracterización. Dentro de las similitudes, se pueden citar las siguientes:

- Los contratistas encuestados tienen un radio de acción acotado al ámbito de los partidos o departamentos en los cuales residen.

- Predominan las formas jurídicas simples.

- El modo de vinculación con los usuarios de sus servicios está atravesado por vínculos basados en el conocimiento previo y la confianza mutua.

- Como resultado de las inversiones con fondos provenientes de distintas fuentes, los parques de maquinarias pueden considerarse modernos y actualizados.

- No participan de tipo alguno de forma asociativa. Esto podría indicar la inexistencia o debilidad de formas asociativas de los propios contratistas, como resultado del predominio del trabajo individual y de sus limitaciones para explorar y experimentar alternativas organizativas que, en muchas situaciones, implicaría la ruptura de concepciones individualistas.

Las diferencias registradas en este estudio permiten delimitar dos tipos categorizados, según la relevancia que adquiere la producción agropecuaria y la prestación de servicios dentro de su estrategia económica y productiva. Aquellos que consideran como actividad principal la producción agropecuaria $(\mathrm{PC})$, privilegian la expansión de la superficie operada por cuenta propia, priorizan la utilización de la maquinaria dentro de sus establecimientos, prestan servicios diversificados y la mano de obra empleada es predominantemente familiar.

En su trabajo sobre los contratistas de un partido (Pehuajó) de la provincia de Buenos Aires, Moreno (2011), destaca la incidencia de la familia en la organización del trabajo y de la empresa. La autora manifiesta que en numerosos casos la prestación de servicios de maquinaria es una actividad que los padres ceden a los hijos, a la cual incorporan inicialmente como empleados para convertirlos posteriormente en socios.

Por su parte, para quienes tienen como actividad principal la prestación de servicios (CP), la superficie trabajada para terceros supera ampliamente la operada por cuenta propia, se especializan en las labores asociadas al modelo tecnológico vigente y la fuerza de trabajo es preponderantemente asalariada.

Algunos de ellos prestan servicios casi en forma exclusiva a ciertos clientes cuya característica es su gran escala de producción. Con este tipo de vínculo, pueden encubrir una relación de dependencia con un empleador en lugar de ser efectuada bajo la modalidad de cuentapropismo (Moreno, 2011).

Una última reflexión, consiste en la posibilidad de vincular la figura del contratista a un proceso de concentración de la producción, dado que la indivisibilidad del capital invertido en tecnología mecánica incentiva el aumento de escala e incide en los cambios en la estructura agraria pampeana. Asimismo, De Martinelli (2008) sostiene que como bien constituye, en cierta medida, una síntesis de las nuevas formas sociales de producción.

\section{Referencias}

Agüero, R., Rivarola, A., \& Maldonado, R. (2007). Caracterización del contratismo de servicios en un sector de la pampa cordobesa: las localidades de Alcira Gigena y Berrotarán. Revista de Estudios Agrarios, 7(14), 1-23.

Azcuy-Ameghino, E., \& Fernández, D. (Abril, 2008). Causas, mecanismos, problemas y debates en torno al proceso de concentración del capital agrario en la región pampeana. Trabajo presentado en la V Jornada de Investigación y Debate: Tra- 
bajo, propiedad y tecnología en el mundo rural argentino, Buenos Aires-Argentina.

Beltrán, C. (2007). Difusión de contratos y aparición de "big players" en el sector agropecuario argentino. Algunos casos. Recuperado de http://goo.gl/EYm9fX

Bustamante, M., \& Maldonado, G. (2008). Actores sociales en el agro pampeano argentino hoy. Algunos aportes para su tipificación. Cuadernos Geográficos, 44(1), 171-191.

Bustillo, M. (2004). Los contratistas del partido de Pergamino a fines de la década de los noventa (Tesis de grado inédita). Universidad de Buenos Aires, Buenos Aires-Argentina.

De Martinelli, G. (2008). Pools de siembra y contratistas de labores. Nuevos y viejos actores sociales en la expansión productiva pampeana reciente. En J. Balsa, G. Mateo \& M. S. Ospital (Comps.), Pasado y presente en el agro argentino (pp. 547-570). Argentina: Editorial Lumiere.

De Nicola, M. (Noviembre, 2003). Cambios en el modelo tecnológico de la producción familiar en la región pampeana en los noventa. Trabajo presentado en la III Jornada Interdisciplinaria de Estudios Agrarios y Agroindustriales, Buenos Aires-Argentina.

Gallo-Mendoza, G. (1970). Definición de reformas agrarias y reforma agraria en Argentina. En E. Flores (Ed.), Reformas Agrarias en América Latina (pp.324-346). Argentina: Juárez Editor.

Garbers R. (Noviembre, 2009). Contratistas de Agromaquinaria: fortaleza del agro Argentino. Trabajo presentado en la VI Jornada Interdisciplinaria de Estudios Agrarios y Agroindustriales, Buenos Aires-Argentina.

González, M. C., Román M., \& Blanchard, G. (Noviembre, 2001). Los contratistas de maquinaria agricola en el partido de Azul, provincia de Buenos Aires. Trabajo presentado en la II Jornadas Interdisciplinarias de Estudios Agrarios y Agroindustriales, Buenos Aires-Argentina.

Lódola, A. (2008). Contratistas, cambios tecnológicos y organizacionales en el agro argentino (Documento de Proyecto No 24). Santiago de Chile: CEPAL.

Moreno, M. (Noviembre, 2011). Estructura social agraria pampeana. Contratistas de labores y vinculos sociales en el partido de Pehuajó. Trabajo presentado en la VII Jornadas Interdisciplinarias de Estudios Agrarios y Agroindustriales, Buenos Aires-Argentina.

Muzlera, J. (Noviembre, 2010). Los contratistas de servicios agropecuarios. Historias productivas y estrategias de capitalización. Trabajo presentado en el VIII Congreso Latinoamericano de Sociología Rural, Porto de Gallinas-Brasil.

Piñeiro, M., \& Villarreal, F. (2005). Modernización agrícola y nuevos actores sociales. Ciencia Hoy, 15(87), 32-36.

Preda, G. (2000). ¿Productores accidentales o empresarios flexibles? Lógicas económicas y organización social de la producción entre contratistas del sudeste de Córdoba. Realidad Económica, 172, 139-156.

Pucciarelli, A. (1998). Cambios en la estructura agraria de la pampa bonaerense (1960-1988). Revista Ciclos, 5, 69-91.

Retamoza, C. (Noviembre, 2001). Contratistas accidentales en una zona agricola de la provincia de Entre Rios (departamentos Paraná, Nogoyá, Victoria y Diamante). Trabajo presentado en la II Jornada Interdisciplinaria de Estudios Agrarios y Agroindustriales, Buenos Aires-Argentina.

Slutzky, D. (2009). Los cambios en la tenencia de la tierra en el pais con especial referencia a la región pampeana: nuevos y viejos actores sociales. Trabajo presentado en la VI Jornada Interdisciplinaria de Estudios Agrarios y Agroindustriales, Buenos Aires-Argentina.

Tort, M. I. (1983). Los contratistas de maquinaria agricola: una modalidad de organización económica del trabajo agrícola en la pampa húmeda (Documento de Trabajo No 11). Buenos Aires: CEIL

Villulla, J. M., \& Amarilla, C. (Noviembre, 2011). ¿Qué es un contratista? Una síntesis de los estudios sobre su historia y un intento de caracterización social. Trabajo presentado en la VII Jornada Interdisciplinaria de Estudios Agrarios y Agroindustriales, Buenos Aires-Argentina.

Zulian, S. B. (2010). Aparición de nuevas estructuras organizacionales en la Producción Agropecuaria Argentina ante los cambios ocurridos en la transformación de la estructura social y la concentración del capital. Revista Agromensajes, 29(9), Recuperado de http://goo.gl/lHMroo 